\title{
Is it time to introduce anti-inflammatory drugs into secondary cardiovascular prevention: evidence from clinical trials?
}

\author{
Aldo Pietro Maggioni ${ }^{1,2}$, Adelaide lervolino ${ }^{3}$, Felicita Andreotti ${ }^{3,4}$ \\ ${ }^{1}$ ANMCO Research Center, Fondazione per il Tuo cuore, Florence 50121, Italy. \\ ${ }^{2}$ GVM Care \& Research, Maria Cecilia Hospital, Cotignola 48010, Italy. \\ ${ }^{3}$ Catholic University of the Sacred Heart, Rome 00168, Italy. \\ ${ }^{4}$ Fondazione Policlinico Universitario A. Gemelli IRCCS, Rome 00168, Italy.
}

Correspondence to: Dr. Aldo Pietro Maggioni, ANMCO Research Centre, Heart Care Foundation, Via La Marmora, 36-Florence, 50121, Italy. E-mail: maggioni@anmco.it

\begin{abstract}
How to cite this article: Maggioni AP, lervolino A, Andreotti F. Is it time to introduce anti-inflammatory drugs into secondary cardiovascular prevention: evidence from clinical trials? Vessel Plus 2021;5:xx. http://dx.doi.org/10.20517/2574-1209.2021.05
\end{abstract}

Received: 12 Jan 2021 First Decision: 18 Jan 2021 Revised: 19 Jan 2021 Accepted: 26 Jan 2021 Published: 12 Mar 2021

Academic Editor: Mario F.L. Gaudino Copy Editor: Yue-Yue Zhang Production Editor: Xi-Jun Chen

\begin{abstract}
A relationship between inflammatory activity, on the one hand, and haemostasias, cardiovascular risk factors and multiple phases of atherothrombotic diseases, on the other hand, has been documented for decades, but only recently have four large trials tested whether anti-inflammatory drugs could prevent major cardiovascular events (MACE) in > 25,000 patients followed on average for 1.9-3.7 years. In patients with recent myocardial infarction $(\mathrm{MI})$ and serum C-reactive protein (CRP) $\geq 2 \mathrm{mg} / \mathrm{L}$, the CANTOS trial showed that subcutaneous three-monthly $300 \mathrm{mg}$ canakinumab [a high-cost, monoclonal antibody to interleukin (IL)-1 $\beta$ ] reduced MACE versus placebo, but it increased fatal infections. In patients with recent MI (COLCOT trial) and in patients with chronic coronary syndromes (LoDoCo2 trial), oral $0.5 \mathrm{mg}$ daily colchicine [a low-cost inhibitor of nucleotide-binding oligomerization domain (NOD)-like receptor (NLR) family pyrin domain containing protein 3 (NLRP3) inflammasome] reduced MACE compared to placebo, with a small but significant increase in pneumonia $(0.9 \%$ vs. $0.4 \%$ in COLCOT; not confirmed in LoDoCo2). In coronary artery disease patients with type 2 diabetes or metabolic syndrome, the CIRT trial found that oral 15-20 mg weekly methotrexate (an anti-rheumatic drug with multiple effects including inhibition of nuclear factor $\mathrm{kB}$ activity) compared to placebo did not reduce MACE, nor circulating IL-6 or CRP, while increasing the risk of non-basal cell skin cancer. Thus, three out of four large trials have now proven that drugs inhibiting the IL-1/IL-6 inflammatory axis can prevent MACE in patients with coronary artery disease. Colchicine, given its overall profile, is likely to become an integral part of secondary cardiovascular-disease prevention strategies.
\end{abstract}

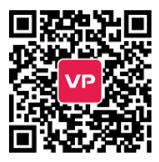


Keywords: Inflammation, atherosclerosis, thrombosis, cardiovascular events, colchicine, canakinumab, methotrexate

\section{INTRODUCTION: INFLAMMATION IN CARDIOVASCULAR DISEASE}

Inflammation characterizes at least six aspects of cardiovascular disease. First, an acute-phase response occurs immediately after the onset of myocardial infarction (MI), with peak plasma C-reactive protein (CRP) values and the duration of CRP elevation related to the extent of infarction ${ }^{[1-3]}$. Second, subclinical low-grade inflammation increases the risk of cardiovascular events; seminal epidemiological studies first showed this, with raised levels of plasma fibrinogen or white blood cell count within the normal range predicting cardiovascular events ${ }^{[4-6]}$, and then Maseri's group reported that CRP levels could predict adverse outcomes in patients with unstable angina ${ }^{[7]}$. Third, atherosclerosis itself can be defined as a chronic inflammatory disease ${ }^{[8]}$. Fourth, acquired cardiovascular risk factors are related to subclinical inflammation in a graded way: the more risk factors, the greater the levels of $\mathrm{CRP}^{[9]}$. Fifth, flares of vascular and atherosclerotic plaque inflammation underlie acute coronary syndromes and run hand in hand with plaque instability and its thrombotic complications ${ }^{[10,11]}$. Sixth, inflammatory cytokines induce hepatic and endothelial-cell synthesis and secretion of prothrombotic factors ${ }^{[3]}$. On this broad and longstanding background, inflammation has grown to be considered a potential therapeutic target for the secondary prevention of atherothrombotic events ${ }^{[10,12,13]}$, involving in particular the interleukin (IL)-1/ IL-6/CRP axis ${ }^{[9,14]}$. Only recently, however, have four large scale studies tested whether treatment with antiinflammatory drugs, approved for other diseases, could prevent atherothrombotic cardiovascular events.

\section{MAJOR RANDOMIZED PLACEBO-CONTROLLED TRIALS}

\section{The canakinumab anti-inflammatory thrombosis outcomes study ${ }^{[15]}$}

The Canakinumab Anti-inflammatory Thrombosis Outcomes Study (CANTOS) was a double-blind trial of over 10,000 patients followed for a mean of 3.7 years. It tested canakinumab, a monoclonal antibody against IL-1 $\beta$ already approved to treat rheumatic disorders, at three doses (50, 150 or $300 \mathrm{mg}$ subcutaneously every three months) against placebo. Enrolled subjects had a history of MI, a blood level of CRP $\geq 2 \mathrm{mg} / \mathrm{L}$ and otherwise well controlled risk factors. At the highest dose (300 mg every three months), canakinumab versus placebo significantly reduced plasma IL- 6 and CRP and the combined endpoint of cardiovascular death, nonfatal MI and nonfatal stroke compared to placebo: 3.90 vs. 4.50 events per 100 person-years [hazard ratio 0.86; 95\% confidence interval (CI) 0.75-0.99; $P=0.031$ ]. The two other tested doses did not provide favorable results. With respect to safety, canakinumab was associated with a higher incidence of fatal infection than was placebo, while there was no significant difference in terms of all-cause mortality.

Although canakinumab did not enter the cardiovascular therapeutic arena, mainly for its high cost and potential risk of sepsis (in some cases fatal), the study was extremely relevant in defining the key role of the IL-1/IL-6/CRP cascade in the genesis of atherothrombotic events. The continued search for an economically sustainable, reasonably safe and feasible large-scale treatment led to two different pharmacological approaches being tested in ad hoc trials: low-dose methotrexate (CIRT) ${ }^{[16]}$ and low-dose colchicine (COLCOT ${ }^{[17]}$ and $\operatorname{LoDoCo} 2^{[18]}$ ).

\section{The cardiovascular inflammation reduction trial ${ }^{[16]}$}

The cardiovascular inflammation reduction trial (CIRT) was a randomized, National Institutes of Healthsponsored, double-blind trial of low-dose methotrexate (target dose 15-20 mg once weekly) or matching placebo conducted in 4786 patients with previous MI or documented multivessel coronary disease who additionally had either type 2 diabetes or metabolic syndrome. Patients were followed for a median of 2.3 years. The primary endpoint was the classical triple major adverse cardiovascular events (MACE), i.e., a 
composite of nonfatal MI, nonfatal stroke or cardiovascular death. Hospitalization for unstable anginadriven revascularization was subsequently added, given a lower than originally assumed rate of events.

The results of the trial were disappointing: low-dose methotrexate did not reduce circulating levels of IL-1 $\beta$, IL-6 or CRP, nor cardiovascular events susver placebo (201 vs. 207; hazard ratio 0.96; 95\%CI: 0.79-1.16). Mouth sores, leukopenia, non-intentional weight loss and transaminase elevations were more common with methotrexate. Cancer was also more frequent with methotrexate versus placebo (52 vs. 30; $P=0.02$ ), mainly due to non-basal-cell skin cancer. Based on these findings, the trial was terminated early for futility.

\section{The colchicine cardiovascular outcomes trial ${ }^{[17]}$}

The colchicine cardiovascular outcomes trial (COLCOT) was an independent, Canadian governmentfunded, multinational, randomized, double-blind trial of 4745 patients with a MI occurring within 30 days of randomization, followed for a median of 2.3 years. Patients, regardless of CRP values and treated with optimal secondary prevention therapies, received colchicine ( $0.5 \mathrm{mg}$ once daily) or placebo. The primary endpoint was a combination of cardiovascular death, resuscitated cardiac arrest, nonfatal MI or stroke and unstable angina-driven revascularization. The primary endpoint was documented in $5.5 \%$ receiving colchicine compared to $7.1 \%$ receiving placebo, a significant $23 \%$ reduction (hazard ratio 0.77 ; $95 \% \mathrm{CI}$ : 0.61 0.96; $P=0.02$ ). Treatment with colchicine determined a favorable effect on each component of the primary endpoint. All-cause mortality did not differ by treatment (43 deaths with colchicine $v s .44$ deaths with placebo). With respect to tolerability/safety, diarrhea was reported in $9.7 \%$ on colchicine versus $8.9 \%$ on placebo, a statistically nonsignificant difference. Pneumonia was recorded more frequently with colchicine versus placebo, albeit in the context of a low incidence of this event ( $0.9 \%$ colchicine $v$ s. $0.4 \%$ placebo; $P=0.03$ ). A study limitation was the relatively short follow-up and the limited number of patients, sufficient to give reliable responses in the overall study population but not in specific subgroups.

COLCOT confirmed the key role of inflammation in the progression of coronary heart disease and offered an effective, low-cost, reasonably safe, preventive treatment, already known to the medical community, given colchicine's indication for gout, familial Mediterranean fever and pericarditis. The results of COLCOT cannot be generalized to all patients with documented coronary artery disease, because they are limited to patients with a recent MI, in whom the intensity of inflammation is likely more relevant. An open question was whether colchicine would be able to prevent ischemic events in stable subjects who had had a MI long before or had documented coronary artery disease but no previous acute event.

\section{The LOw-DOse COlchicine 2 study $^{[18]}$}

The LOw-DOse COlchicine 2 (LODOCO2) was a randomized, placebo-controlled, double-blind study that included 5500 patients with documented coronary artery disease who had been stable for at least six months; patients were followed for a median of 2.4 years. The study was conducted in Australia and the Netherlands and was funded by public and private foundations and a consortium of pharmaceutical industries. Patients were randomized to low-dose colchicine ( $0.5 \mathrm{mg}$ per day) or placebo. The primary endpoint was a composite of cardiovascular death, spontaneous (non-periprocedural) MI, ischemic stroke or ischemia-driven revascularization. The main secondary endpoint was the classic MACE (cardiovascular death, nonfatal MI or nonfatal stroke). Again, the results were favorable. The primary endpoint occurred in $6.8 \%$ with colchicine versus $9.6 \%$ with placebo, a highly significant $31 \%$ relative risk reduction (hazard ratio $0.69 ; 95 \%$ CI: $0.57-0.83 ; P<0.001)$. The main secondary endpoint was reduced by $28 \%(4.2 \%$ on colchicine vs. $5.7 \%$ on placebo; hazard ratio $0.72 ; 95 \% \mathrm{CI}$ : $0.57-0.92 ; P=0.007)$, which should be considered significant not only from a statistical point of view but also for clinical relevance. Regarding safety, there were no significant differences in expected adverse reactions compared to placebo, not even regarding pneumonia or gastrointestinal disorders. The incidence of non-cardiovascular death was nominally higher (but not statistically significant) with colchicine versus placebo ( 0.7 vs. 0.5 events per 100 person-years; hazard 


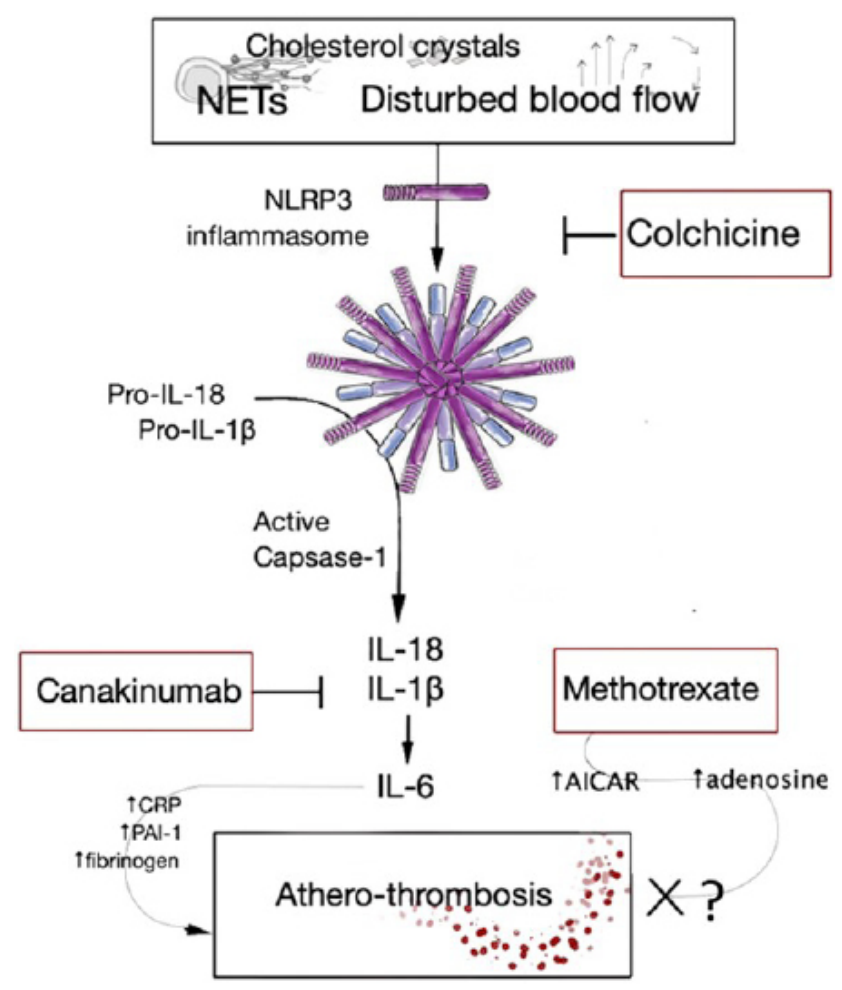

Figure 1. Inflammatory signaling inhibited by colchicine, canakinumab and methotrexate. The NLRP3 inflammasome is inhibited upstream to its assembly by colchicine, whereas canakinumab acts downstream, after caspase-1-dependent cleavage of pro-IL-1 13 . Methotrexate has multiple effects including inhibition of AICAR transformylase, leading to AICAR accumulation and adenosine release at inflamed sites. AICAR: 5-aminoimidazole-4-carboxamide ribonucleotide; CRP: C reactive protein; IL: interleukin; NET: neutrophil extracellular traps; NLRP3: nucleotide-binding oligomerization domain(NOD)-like receptor (NLR) family pyrin domain containing protein 3 (NLRP3); PAI-1: plasminogen activator inhibitor type 1.

ratio 1.51; 95\%CI: 0.99-2.31). Subgroup analyses showed homogeneous effects in all analyzed subgroups; however, an important limitation of the study is the lack of information on the levels of inflammatory markers (e.g., CRP) at baseline and after treatment.

Thus, LoDoCo2 and COLCOT seem to close the circle by confirming that inflammation is one of the potential determinants of coronary heart disease progression and that an anti-inflammatory drug can favorably prevent new vascular events in those presenting a recent MI (COLCOT) or a more chronic coronary syndrome (LoDoCo2).

\section{MECHANISMS OF ACTION OF CANAKINUMAB, COLCHICINE AND METHOTREXATE}

Suppression of the inflammatory response by the above drugs occurs at different levels [Figure 1]. Canakinumab is a recombinant anti-IL- $1 \beta$ monoclonal antibody of the immunoglobulin $\mathrm{G} 1 / \kappa$ subclass. It neutralizes the inflammatory signaling induced by the IL-1 $\beta$ interaction with IL-1 receptors on lymphoid, myeloid, endothelial and other cell types ${ }^{[15,19]}$; IL-1 $\beta$ is a key mediator of Th17 development ${ }^{[20]}$. Colchicine is a plant-derived alkaloid that reduces the synthesis of IL-1 $\beta$, IL-2 and IL- 6 by inhibiting tubulin polymerization and consequently the nucleotide-binding oligomerization domain (NOD)-like receptor (NLR) family pyrin domain containing protein 3 (NLRP3) inflammasome arrangement in monocytes and other cells ${ }^{[21,22]}$. Colchicine is also reported to downregulate neutrophil adhesion and recruitment, superoxide anion production and mast cell degranulation ${ }^{[21,22]}$ and to inhibit cholesterol-crystal induced endothelial pyroptosis, oxidative stress and monocyte chemotaxis ${ }^{[23]}$, as well as platelet aggregation and endothelial cell prothrombotic effects induced by oxidized $\mathrm{LDL}^{[24,25]}$. In an animal model, colchicine 


\section{Secondary prevention strategies for patients with documented chronic coronary syndrome}

Changes in life style (smoking cessation, body weight control, diet, exercise),

SBP ( $<130 \mathrm{mmHg}$ ) and $\mathrm{HbA1c}$ in diabetics $(<6.5 \%)$

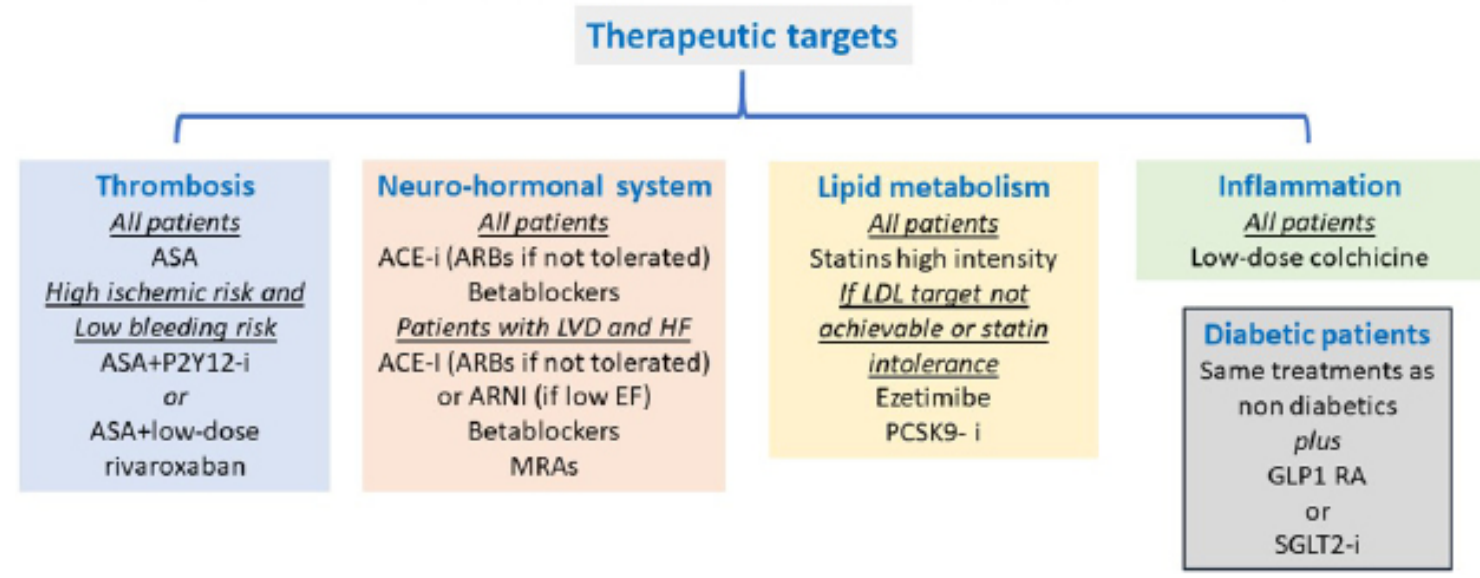

Figure 2. Secondary prevention strategies for patients with documented chronic coronary syndromes. Anti-inflammatory therapy will likely be included in future guidelines. ACE: Angiotensin converting enzyme; ARB: angiotensin II receptor blocker; ASA: aspirin; EF: ejection fraction; GLP1 RA: glucagon-like peptide-1 receptor agonist; Hb: hemoglobin; HFP: heart failure; i: inhibitor; LDL: low density lipoprotein; LVD: left ventricular dysfunction; PCSK9: proprotein convertase subtilisin/kexin type 9; SBP: systolic blood pressure; SGLT2: sodium-glucose cotransporter-2.

protected against acute cerebral ischemia by inhibiting exocytosis and chemotaxis ${ }^{[26]}$. Methotrexate is a disease-modifying anti-rheumatic drug with multiple effects, including inhibition of nuclear factor $\kappa \mathrm{B}$ $\operatorname{activity}^{[27]}$, and is used in chemotherapy as inhibitor of dihydrofolate reductase and, consequently, of DNA/ RNA synthesis; this drug also increases intracellular and extracellular levels of adenosine ${ }^{[27]}$. Nonetheless, its administration in humans failed to reduce circulating IL-1 $\beta$, IL-6 and CRP levels and the occurrence of cardiovascular events ${ }^{[16]}$.

\section{CLINICAL AND RESEARCH IMPLICATIONS}

\section{Updated secondary prevention in clinical practice}

CANTOS, COLCOT and LoDoCo2 expand the scenario of secondary prevention strategies for coronary heart disease. CANTOS proved the strong involvement of the inflammatory cytokine IL-1 $\beta$ in atherothrombotic events, and colchicine confirmed the benefit of inhibiting inflammation in patients with recent MI or chronic coronary syndromes. Inflammation therefore might be added to the three traditional therapeutic targets (thrombosis, lipid metabolism and neuro-hormonal system) [Figure 2]. It is very likely that forthcoming updated versions of international guidelines will contain indications on the use of the anti-inflammatory drug colchicine for secondary prevention purposes. The addition of a new drug on top of existing ones will rekindle the problem of adherence to recommended treatments for both prescribers and patients, and it may encourage possible de-prescribing (in this context, for example, the role of betablockers for all patients with chronic coronary syndrome is currently debated ${ }^{[28,29]}$ ). It will be necessary to plan strategies aimed not only at producing the best possible knowledge of recommended treatments for both healthcare professionals and patients, but, even more, at improving clinical implementation, possibly through better communication of healthcare protocols between hospitals and the community. 


\section{Research perspectives}

Research on the role of inflammation and of anti-inflammatory strategies (i.e., drugs, dietary habits, exercise and lifestyle changes) in atherothrombotic diseases is continuing intensely with $>220$ interventional studies reported on clinicaltrial.gov ${ }^{[30]}$. The favorable results of the colchicine coronary heart disease trials and the interest in a low-cost but highly effective anti-inflammatory treatment is stimulating research in other cardiovascular areas and beyond, with $>170$ controlled colchicine studies registered on clinicaltrial.gov in diverse conditions, including prevention or delay of: (1) new cerebrovascular events in patients with a previous stroke or TIA (CONVINCE) ${ }^{[31]}$; (2) nephropathy progression in patients with diabetes mellitus and microalbuminuria ${ }^{[32]}$; (3) mortality; and (4) major respiratory complications in hospitalized patients (COLCOVID) ${ }^{[33]}$ and outpatients (COLCORONA) $)^{[34]}$ with Coronavirus Disease 2019 (COVID-19).

\section{CONCLUSIONS}

Recent large controlled trials have confirmed the role of inflammation in the pathogenesis of atherothrombotic disorders and expanded treatment options in patients with coronary heart disease. A relevant change in secondary prevention strategies is taking place with the inclusion of drugs targeted against inflammation. In this context, the revival of an old drug, colchicine, is likely to enrich our therapeutic toolbox, beyond the well-known clinical indications of gout and pericarditis ${ }^{[35]}$, and with possible applications to different clinical areas in which inflammation may play a relevant role, including COVID-19, a condition in which reliable results from well-conducted studies demonstrating effective therapies are desperately needed.

\section{DECLARATIONS}

\section{Authors' contributions}

All authors contributed equally to the critical evaluation and whole content of the manuscript.

Conceptualized and prepared the original draft: Maggioni AP, Andreotti F

Drafted and revised figures: Maggioni AP, Iervolino A, Andreotti F

Reviewed and edited the manuscript: Maggioni AP, Iervolino A, Andreotti F

\section{Availability of data and materials}

Not applicable.

\section{Financial support and sponsorship}

None.

\section{Conflict of interest}

Maggioni AP received personal fees from Bayer, Fresenius, Novartis for the participation in study committees outside the present work. Iervolino A reports no conflicts of interest to disclose. Andreotti F reports receiving personal fees from Amgen, Bayer, BMS-Pfizer and Daiichi Sankyo outside the present work.

\section{Ethical approval and consent to participate}

Not applicable.

\section{Consent for publication}

Not applicable.

\section{Copyright}

(c) The Author(s) 2021. 


\section{REFERENCES}

1. Kroop IG, Shackman NH. Level of C-reactive protein as a measure of acute myocardial infarction. Proc Soc Exp Biol Med 1954;86:95-7.

2. Pepys MB, Baltz ML. Acute phase proteins with special reference to C-reactive protein and related proteins (pentraxins) and serum amyloid A protein. Adv Immunol 1983;34:141-212.

3. Andreotti F, Roncaglioni MC, Hackett DR, el al. Early coronary reperfusion blunts the procoagulant response of plasminogen activator inhibitor-1 and von Willebrand factor in acute myocardial infarction. J Am Coll Cardiol 1990;16:1553-60.

4. Meade TW, Mellows S, Brozovic M, et al. Haemostatic function and ischaemic heart disease: principal results of the Northwick Park Heart Study. Lancet 1986;2:533-7.

5. Kannel WB. Fibrinogen and risk of cardiovascular disease: the framingham study. JAMA 1987;258:1183.

6. Yarnell JW, Baker IA, Sweetnam PM, et al. Fibrinogen, viscosity, and white blood cell count are major risk factors for ischemic heart disease. The Caerphilly and Speedwell collaborative heart disease studies. Circulation 1991;83:836-44.

7. Liuzzo G, Biasucci LM, Gallimore JR, et al. The prognostic value of C-reactive protein and serum amyloid a protein in severe unstable angina. N Engl J Med 1994;331:417-24.

8. Ross R. Atherosclerosis-an inflammatory disease. N Engl J Med 1999;340:115-26.

9. Bermudez EA, Rifai N, Buring J, Manson JE, Ridker PM. Interrelationships among circulating interleukin-6, C-reactive protein, and traditional cardiovascular risk factors in women. Arterioscler Thromb Vasc Biol 2002;22:1668-73

10. Kohchi K, Takebayashi S, Hiroki T, Nobuyoshi M. Significance of adventitial inflammation of the coronary artery in patients with unstable angina: results at autopsy. Circulation 1985;71:709-16.

11. Crea F, Andreotti F. The unstable plaque: a broken balance. Eur Heart J 2009;30:1821-3.

12. Hansson GK. Inflammation, atherosclerosis, and coronary artery disease. N Engl J Med 2005;352:1685-95.

13. Libby P, Ridker PM, Hansson GK; Leducq Transatlantic Network on Atherothrombosis. Inflammation in atherosclerosis: from pathophysiology to practice. J Am Coll Cardiol 2009;54:2129-38.

14. Morton AC, Rothman AM, Greenwood JP, et al. The effect of interleukin-1 receptor antagonist therapy on markers of inflammation in non-ST elevation acute coronary syndromes: the MRC-ILA Heart Study. Eur Heart J 2015;36:377-84.

15. Ridker PM, Everett BM, Thuren T, et al; CANTOS Trial Group. Antiinflammatory therapy with canakinumab for atherosclerotic disease. N Engl J Med 2017;377:1119-31.

16. Ridker PM, Everett BM, Pradhan A, et al; CIRT Investigators. Low-dose methotrexate for the prevention of atherosclerotic events. $N$ Engl J Med 2019;380:752-62.

17. Tardif JC, Kouz S, Waters DD, et al. Efficacy and safety of low-dose colchicine after myocardial infarction. N Engl J Med 2019;381:2497505.

18. Nidorf SM, Fiolet ATL, Mosterd A, et al; LoDoCo2 Trial Investigators. Colchicine in Patients with Chronic Coronary Disease. $N$ Engl $J$ Med 2020;383:1838-47.

19. Buckley LF, Abbate A. Interleukin-1 blockade in cardiovascular diseases: a clinical update. Eur Heart J 2018;39:2063-9.

20. Mailer RK, Joly AL, Liu S, Elias S, Tegner J, Andersson J. IL-1 $\beta$ promotes Th17 differentiation by inducing alternative splicing of FOXP3. Sci Rep 2015;5:14674.

21. Dalbeth N, Lauterio TJ, Wolfe HR. Mechanism of action of colchicine in the treatment of gout. Clin Ther 2014;36:1465-79.

22. El Hasbani G, Jawad A, Uthman I. Colchicine: An ancient drug with multiple benefits. Curr Pharm Des 2020.

23. Yang M, Lv H, Liu Q, et al. Colchicine alleviates cholesterol crystal-induced endothelial cell pyroptosis through activating AMPK/SIRT1 pathway. Oxid Med Cell Longev 2020;2020:9173530.

24. Cirillo P, Taglialatela V, Pellegrino G, et al. Effects of colchicine on platelet aggregation in patients on dual antiplatelet therapy with aspirin and clopidogrel. $J$ Thromb Thrombolysis 2020;50:468-72.

25. Cimmino G, Conte S, Morello A, et al. Colchicine inhibits the prothrombotic effects of oxLDL in human endothelial cells. Vascul Pharmacol 2020;:106822.

26. Pratt J, Roux M, Henneguelle E, Stutzmann JM, Laduron PM. Neuroprotective effects of colchicine in the gerbil model of cerebral ischaemia. Neurosci Lett 1994;169:114-8.

27. Bedoui Y, Guillot X, Sélambarom J, et al. Methotrexate an old drug with new tricks. Int J Mol Sci 2019;20:5023.

28. Ziff OJ, Samra M, Howard JP, et al. Beta-blocker efficacy across different cardiovascular indications: an umbrella review and metaanalytic assessment. BMC Med 2020;18:103.

29. Bangalore S, Makani H, Radford M, et al. Clinical outcomes with $\beta$-blockers for myocardial infarction: a meta-analysis of randomized trials. Am J Med 2014;127:939-53.

30. ClinicalTrails.Gov. Available from: https://www.clinicaltrials.gov/ct2/results?term=coronary+disease\&cond=Inflammation\&age_v=\&gnd $\mathrm{r}=\&$ type $=$ Intr\&rslt=\&Search=Apply [Last accessed on 15 Nov 2020]

31. CONVINCE Trial. Available from: https://clinicaltrials.gov/ct2/show/NCT02898610 [Last accessed on 15 Nov 2020]

32. Chinese study. Available from: https://clinicaltrials.gov/ct2/show/NCT02035891 [Last accessed on 15 Nov 2020]

33. COLCOVID Trial. Available from: https://clinicaltrials.gov/ct2/show/NCT04328480 [Last accessed on 15 Nov 2020]

34. COLCORONA Trial. Available from: https://clinicaltrials.gov/ct2/show/NCT04322682 [Last accessed on 15 Nov 2020]

35. Imazio M, Brucato A, Cemin R, et al.; ICAP Investigators. A randomized trial of colchicine for acute pericarditis. $N$ Engl J Med 2013;369:1522-8. 